\title{
Knee Pain During Islamic Prayer after Anterior Cruciate Ligament Reconstruction Using Hamstring and Bone Patellar Tendon Autograft
}

\author{
Ahmed N., Shihabudin M.T., Ab Rahman S. \\ Department of Orthopaedics, Universiti Sains Malaysia
}

\section{ABSTRACT}

Introduction: Hamstring tendon (HT) and bone patellar tendon bone (BPTB) are the commonly used autograft in an anterior cruciate ligament reconstruction surgery. The BPTB is said to have more incidence of anterior knee pain compared to HT. The study aimed to compare the severity of knee pain during Islamic prayer kneeling. Methods: A cross sectional cohort analysis of patients undergoing ACL reconstruction surgery using BPTB with HT autografts was conducted to determine differences in postoperative pain while kneeling and ability to pray in normal position. Kneeling during prayer and the ability to sit while performing prayer were assessed at $3^{\text {rd }}, 4^{\text {th }}, 5^{\text {th }}$ and $6^{\text {th }}$ month. Results: There were no significant differences in mean pain score while kneeling at $3^{\text {rd }}, 4^{\text {th }}, 5^{\text {th }}$ and $6^{\text {th }}$ month. The mean difference of patient's ability to perform normal prayers for BPTB $(3.56+1.16)$ and HT $(3.30+1.05)$ was found insignificant. The average number of patients in both groups was able to pray between 3 to 4 months post operatively as full range of motion of knee is allowed within this period. Total 49 patients (BPTB 23, HT 26) out of 60 were able to pray normally within 4 months post-operation. Mostly delayed due to anterior knee pain. Conclusion: There is no different in term of knee pain during kneeling while performing Islamic prayer between those who had their ACL reconstructed either using BPTB or HT autograft.

KEYWORDS: knee pain, prayer, anterior cruciate ligament, reconstruction

\section{INTRODUCTION}

The two most commonly used autografts during anterior cruciate ligament $(\mathrm{ACL})$ reconstruction are BPTB and hamstrings tendon. ${ }^{1}$ The however the absolute superiority of either of those two most commonly used grafts are debatable..$^{2-5}$ It has been suggested that those who cannot tolerate anterior knee pain due to lifestyle or work involving kneeling should have an HS autograft. ${ }^{6,7}$ Previous literatures have suggested that BTB autograft has a greater risk for chronic anterior knee pain and kneeling pain compared to $\mathrm{HS}^{8,9}$

Normal Muslim prayer involves frequent kneeling that followed by sitting on the floor. There is a possibility

Corresponding author:

Dr. Muzaffar Tengku Shihabudin

Department of Orthopaedics

School of Medical Sciences

Universiti Sains Malaysia

16150 Kubang Kerian, Kelantan, Malaysia.

Tel No: +6097676398

Email : tgmuzaffar@gmail.com that after surgery the knee pain would disturb the kneeling position during prayer and the ability to perform sitting on the floor after kneeling. Studies that assessed the ability to perform normal prayer following $\mathrm{ACL}$ reconstruction using BPTB and $\mathrm{HT}$ among the Muslim community are lacking. It is not known if the knee pain following BPTB graft specifically affects the ability to perform normal Muslim prayer as compared to HT graft. Our study evaluated this aspect and the findings will help medical personnel to discuss with Muslim patients to decide whether BPTB or HT is more suitable for them in terms of prayer kneeling.

The primary aim of this study was to determine whether there was a difference in knee pain while kneeling between those undergoing BPTB and HT graft $\mathrm{ACL}$ reconstruction. Our second aim of this study was to compare the duration of time taken after the surgery before the patient could perform the sitting position (on the floor) after kneeling during praying ritual. We hypothesized that there is no difference in pain level and the duration of time 
taken for a patient to perform normal Muslim prayer following ACL Reconstruction using BPTB as compared to Hamstring tendon.

\section{MATERIALS AND METHODS}

We performed a cross sectional cohort analysis of patients undergoing $A C L$ reconstruction surgery using BTB and HS autograft to determine differences in postoperative pain while kneeling and ability to pray normally. This study was approved by our Human Research Ethic Committee in accordance to the international standard.

From January 2015 to June 2017, consecutive patients treated surgically by a senior Orthopaedics Sports surgeon in our hospital for ACL tears that met the selection criteria were consented for participation. Inclusion criteria were skeletally mature patients older than 18 years till 45 years and patients undergoing primary $A C L$ reconstructions for single knee injury. Exclusion criteria were revision surgery, concurrent other ligamentous injuries requiring repair, present of knee pain during kneeling before surgery and those with existing physical restriction to performed normal prayer position before the surgery.

The surgeon chooses the graft option after a detailed discussion on the risks and benefits of each graft with the patients. The same surgeon treated all patients to increase the consistency of each procedure. For the purpose of this study, we define a normal Muslim prayer when a person is able to perform kneeling follow by sitting on the floor during the praying ritual (figure 1 and 2 ).

Immediately after the surgery, patients were unable to do this maneuver because of the knee range of motion post-operative rehabilitation restriction. After six weeks post-surgery, all patient has no restriction in flexing the knee and that was the reason why we began our assessment after two months post operatively. The points of interest are the knee pain during kneeling (figure 1) and the ability to sit on the floor with the knee flexed (figure 2) immediately after kneeling during the praying ritual. All patients were able to perform these maneuvers before the surgery.

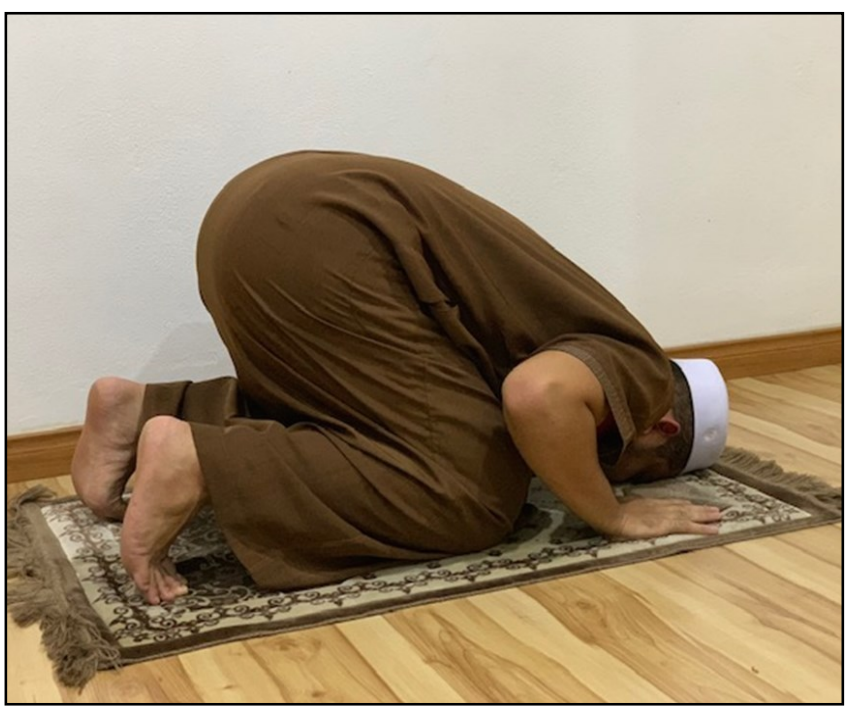

Figure 1: Kneeling position during Islamic prayer

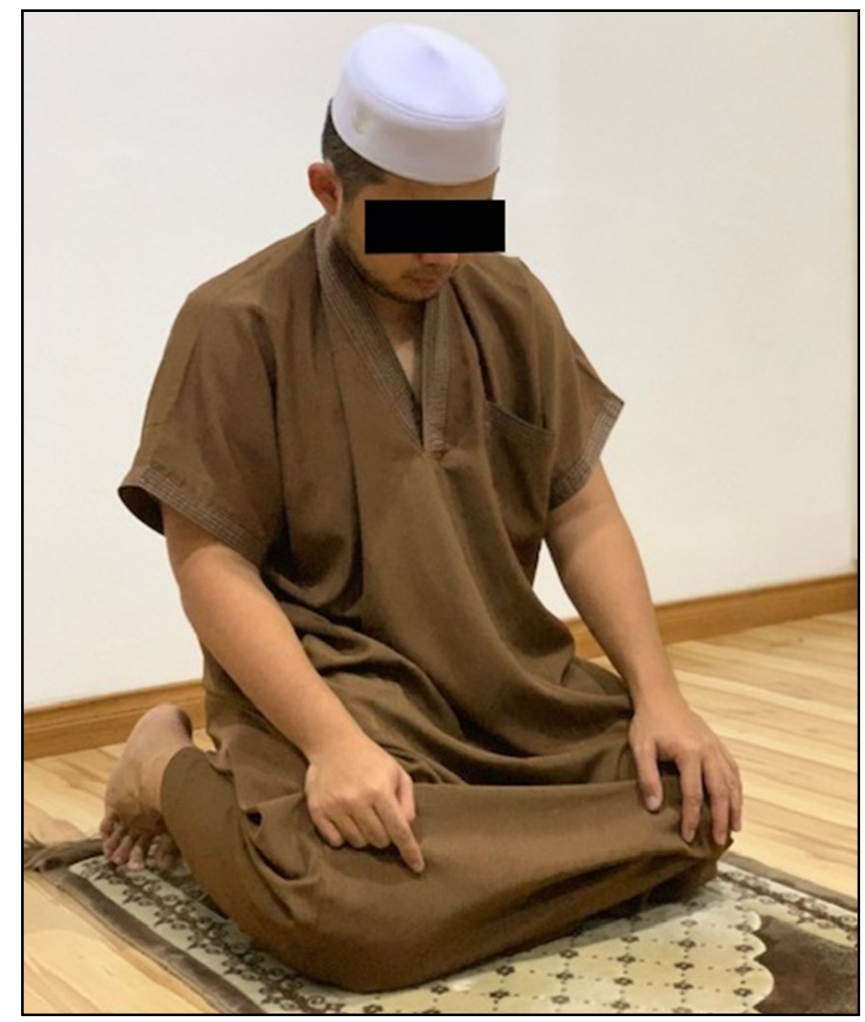

Figure 2: Sitting position after kneeling during Islamic prayer

All patients were followed up at physiotherapy clinic after the operation. Patients underwent the same rehabilitation protocol that we modify from GSLM Anterior Cruciate Ligament Reconstruction rehabilitation $\operatorname{protocol}^{10}$. We allowed full knee flexion at 6-8 weeks post op in the case where meniscal repair was performed. They were assessed if they were able to do kneeling as in the Muslim prayer and they rated the pain score using NRS (Numerical Rating Scale) at $3^{\text {rd }}, 4^{\text {th }}, 5^{\text {th }}$ and $6^{\text {th }}$ month. 
As for the ability to sit on the floor after the kneeling, we record the duration of time taken post operatively before the patient could perform normal prayer. This was confirmed by asking directly the patient or patient performed the maneuver during the assessment. The degree of knee flexion was not measured as it was not our point of interest in this study.

Finally the LYSHOLM Score was assessed at $6^{\text {th }}$ month post-surgery to determine the functional outcome at the endpoint of our study. The score ranges up to 100 (95-100=excellent, 84-94 =good, 65-83=fair, and 64 and below= poor).

\section{Statistical Analysis}

All data were collected and analyzed using SPSS Version 22. They were analyzed using independent 2 group $t$ tests and were reported as means \pm standard deviations. In all analyses, $p<.05$ was considered statistically significant.

\section{RESULTS}

Sixty patients were recruited for the study with both HT and BPTB consist of thirty patients each. Most were male with six female in HT group and one in BPTB group. Demographic differences between the 2 groups are presented in Table 1. All patients had ACL tear during sporting activities. All of them were able to pray in normal position before surgery and did not complain of knee pain during kneeling.

Table I Demographic Data

\begin{tabular}{llll}
\hline & $\begin{array}{l}\text { BPTB } \\
\mathrm{n}=30\end{array}$ & $\begin{array}{l}\mathrm{HT} \\
\mathrm{n}=30\end{array}$ & $\mathrm{p}$ Value \\
\hline $\begin{array}{l}\text { Mean Age } \\
\text { (years) }\end{array}$ & $23.26+03.93$ & $28.26+07.25$ & $.002^{*}$ \\
$\begin{array}{l}\text { Body mass } \\
\text { Index } \\
(\mathrm{Kg} / \mathrm{m} 2)\end{array}$ & $24.19+02.95$ & $25.22+03.56$ & 0.23 \\
$\begin{array}{l}\text { Mean Injury } \\
\text { to Surgery } \\
\begin{array}{l}\text { Interval } \\
\text { (months) }\end{array}\end{array}$ & $11.36+08.21$ & $13.33+11.18$ & 0.37 \\
\hline
\end{tabular}

Data are presented as mean \pm SD. BPTB, bone-patellar tendon-bone; HT, hamstring tendon;

Both groups showed decreased in anterior knee pain from $3^{\text {rd }}$ to $6^{\text {th }}$ month post operatively with no significant differences in mean pain score (Table 2 ). Most patients were able to performed normal prayer before $4^{\text {th }}$ post-operative month (Table 3). Mean duration of patient's ability to perform normal prayers for BPTB and HT was not insignificantly different. All of them were able to perform normal prayers by sixth post-operative month. Both groups demonstrated good Lysholm score at this period $(\mathrm{BPTB}=93.53+7.16, \mathrm{HT}=90.63+9.57)$ and there was no significance difference between these groups $(p=0.19)$.

Table II: Comparison of mean difference of anterior knee pain score while kneeling at $3^{\text {rd }}$ until $6^{\text {th }}$ months after the $A C L$ reconstructive surgery using BPTB and $\mathrm{HT}$ autograft

\begin{tabular}{llcc}
\multicolumn{5}{c}{ Mean Pain Score while kneeling } \\
\hline \multicolumn{3}{c}{ NRS } \\
\hline POM & BPTB & HT & p Value \\
3 & $3.43+1.75$ & $3.63+2.31$ & 0.71 \\
4 & $2.56+1.65$ & $2.66+1.97$ & 0.83 \\
5 & $1.53+1.55$ & $1.90+1.54$ & 0.36 \\
6 & $0.57+1.04$ & $1.07+1.05$ & 0.07
\end{tabular}

$\mathrm{n}=60(\mathrm{BPTB}=30, \mathrm{HT}=30)$. Data are presented as mean $\pm \mathrm{SD}$. $\mathrm{BPTB}$, bone-patellar tendon-bone; $\mathrm{HT}$, hamstring tendon; POM, postoperative Month; NRS, numerical rating scale.

Table III: Comparing the ability to pray normally after the

\begin{tabular}{ccc}
\hline & \multicolumn{2}{c}{ Ability to pray in normal position } \\
\cline { 2 - 3 } POM & BPTB & HT \\
2 - 3 & 5 & 7 \\
$3-4$ & 18 & 19 \\
$4-5$ & 5 & 3 \\
$5-6$ & 2 & 1 \\
\hline n=60 (BPTB=30, HT=30). BPTB, bone-patellar tendon- \\
bone; HT, hamstring tendon; POM, postoperative month; \\
DISCUSSION
\end{tabular}

In our study we reviewed the outcome after two months because those patients with meniscal repair would be restricted from full flexion until after eight weeks post-surgery. Our study showed that the knee pain on kneeling during Muslim prayer was comparable between those who had their ACL reconstructed using bone patella bone autograft and hamstring autograft. There was progressive decrease in intensity of pain in both groups throughout the study period. Our study however is in contrary to many literatures that showed the incidence of anterior knee pain is more common in BPTB graft as compared to $\mathrm{HT} .^{11-13}$ Xie et al conducted a metaanalysis of 22 studies with a total of 1930 patient undergoing $\mathrm{ACL}$ reconstruction. ${ }^{9}$ Their study found patients treated with BPTB autograft had more 
significant long-term anterior knee and kneeling pain when compared with those treated with HS autografts. Additionally, Li et al evaluated outcomes after ACL reconstruction across 9 randomized controlled trials totaling 738 patients. ${ }^{14}$ Their study also concluded BPTB autografts produced significant anterior knee pain and kneeling pain.

It is important to highlight that in our study we assess the knee pain specifically on kneeling during Muslim prayer. The kneeling and body position in our study may not be similar to the kneeling in those studies mentioned above. The reason is because during normal Muslim prayer, the kneeling will involve seven body parts that will be in contact with the floor. These body parts are the forehead, both palm of the hands, both knees and toes of both feet. We believed that because of many body parts are in contact with the floor, the weight from the operated knee could have been shifted to the other six body parts, hence there would be less weight bore by the operated knee that probably leads to lesser degree of knee pain. The patient may be doing this unintentionally as a response to the pain born on the operated knee. This could finally leads to no significant different in pain score in our series.

Nevertheless our study did not measure specifically the weight distributed to both knee, therefore further study may be needed to support this explanation.

Literature among the Muslim community that assess whether BPTB or HT autograft would affect the kneeling during Muslim prayer is lacking and not specific. Abbas et al who conducted the study in Muslim country also found that there was no statistically significant difference between the two groups in the incidence of anterior knee pain and kneeling. ${ }^{15}$ However it was not clear whether the author assessed the incidence of knee pain on kneeling during normal Muslim prayer or not. Another study by Ibrahim et al suggest that HT autograft is preferred for the Muslim community as compared to BPTB because they have to perform kneeling during normal daily prayer. ${ }^{6}$ Unfortunately this study did not assess specifically the knee pain on kneeling during prayer, thus making comparison to our study is inappropriate. We believe that kneeling during Muslim prayer where seven body parts are in contact with the floor is not similar to 'usual' kneeling where the body is in vertical that make both knees bear most of the weight.
The current study also revealed that there was no difference in the duration of time taken for a patient to perform normal Muslim prayer following $A C L$ Reconstruction using BPTB and Hamstring tendon. This finding seems to be in parallel with the finding of kneeling pain during prayer in our cohort. As both groups demonstrate no significance differences in term of knee pain during kneeling, this could partly explained that both groups eventually took almost similar time frame after the surgery before they can performed normal prayer. It is also crucial that the term 'normal Muslim prayer' to be clearly define as there is no specific definition. In our study, beside able to stand, the patient need to be able to performed kneeling as well as able to go into sitting position after kneeling (figure 2). We believe that these two positions are the most difficult to performed early after surgery. Our study however did not measure the actual knee range of motion needed to be in those prayer positions.

Our study also demonstrates that in both groups, knee pain was the major reason for delay in performing normal prayer followed by knee stiffness. Most of them were able to pray within 3 - 4 months post-operative in both groups. All of them were able to pray normally at the end of our study period (6 months post op). We believed the pain and stiffness that most of our patients felt were not obviously related to arthrofibrosis. It could rather be due to our rehabilitation protocol, as we only allow full flexion after 8 weeks or 2 months in those with meniscal repair procedure. Our explanation is that immediately after 8 weeks, when the patient tried to fully flex the knee, the pain is felt due to the 'residual stiffness' as a result of rehabilitation protocol restriction. However, after continuation of physiotherapy, full knee flexion or normal prayer position was achieved. This explained why majority of our patients were able to pray in normal sitting position within three to four months post-surgery. This so called 'residual stiffness' could also explain why the pain score was always similar in comparison through-out the study period between BPTB and HT group. Nevertheless it is beyond the scope of this study to evaluate the arthrofibrosis as the cause of pain or stiffness.

Anterior knee pain does occur after hamstring $\mathrm{ACL}$ reconstruction although the anterior structures of the knee remain intact. ${ }^{16}$ The mechanism for this is not clear, but it is known that the patella-femoral joint can be the source of pain after almost any 
surgery to the knee, even if the patella-femoral extension mechanism is not directly involved. Anterior knee pain after patellar tendon harvesting however, is usually more well localized and palpation would suggest trigger points that are commonly over the inferior pole of the patella or the tibial tuberosity or above the patellar tendon donor site ${ }^{17}$.

\section{Limitations}

The non-randomized nature of the study induces potential bias. There was an age difference between the cohorts, with a greater number of younger patients in the BTB group. Another limitation is confounding factors like meniscus procedure performed concomitantly with $\mathrm{ACL}$ reconstruction that could have affected knee pain scores. However, we minimized this bias by excluding those with knee pain during prayer kneeling before the surgery since our interest after 2 months post-surgery because at this time patient's knee range of motion was not restricted in those who had meniscal repair.

\section{CONCLUSION}

Patients who had $\mathrm{ACL}$ reconstruction with BPTB or HS autograft did not show significant difference in knee pain while prayer kneeling. There was also no clinically significant difference in terms of time frame taken post-operatively to perform Islamic prayers in normal position.

\section{Conflict of interest}

All the authors involved in this study has no conflict of interest.

\section{ACKNOWLEDGEMENT}

The authors gratefully acknowledge the physiotherapy members that were involved in patients' rehabilitation.

\section{REFERENCES}

1. Vaishya R, Agarwal AK, Ingole S, et al. Current trends in anterior cruciate ligament reconstruction: a review. Cureus 2015; 7.

2. Wilson TW, Zafuta MP and Zobitz M. A biomechanical analysis of matched bone-patellar tendon-bone and double-looped semitendinosus and gracilis tendon grafts. The American Journal of Sports Medicine 1999; 27: 202-207.

3. Aune AK, Holm I, Risberg MA, et al. Four-strand hamstring tendon autograft compared with patellar tendon-bone autograft for anterior cruciate ligament reconstruction. The American journal of sports medicine 2001; 29: 722-728.

4. Ejerhed L, Kartus J, Sernert N, et al. Patellar tendon or semitendinosus tendon autografts for anterior cruciate ligament reconstruction? The American journal of sports medicine 2003; 31: 19-25.

5. Laxdal G, Kartus J, Hansson L, et al. A prospective randomized comparison of bonepatellar tendon-bone and hamstring grafts for anterior cruciate ligament reconstruction. Arthroscopy: The Journal of Arthroscopic \& Related Surgery 2005; 21: 34-42.

6. Ibrahim SA-R, Al-Kussary IM, Al-Misfer ARK, et al. Clinical evaluation of arthroscopically assisted anterior cruciate ligament reconstruction: patellar tendon versus gracilis and semitendinosus autograft. Arthroscopy: The Journal of Arthroscopic \& Related Surgery 2005; 21: 412-417.

7. Romanini E, D’Angelo F, De Masi S, et al. Graft selection in arthroscopic anterior cruciate ligament reconstruction. Journal of orthopaedics and traumatology 2010; 11: 211219.

8. Pinczewski LA, Lyman J, Salmon LJ, et al. A 10year comparison of anterior cruciate ligament reconstructions with hamstring tendon and patellar tendon autograft. The American journal of sports medicine 2007; 35: 564-574.

9. Xie X, Xiao Z, Li Q, et al. Increased incidence of osteoarthritis of knee joint after ACL reconstruction with bone-patellar tendon-bone autografts than hamstring autografts: a metaanalysis of 1,443 patients at a minimum of 5 years. European Journal of Orthopaedic Surgery \& Traumatology 2015; 25: 149-159.

10. GLSM ACL Reconstruction Rehabilitation Program. http:/ / wwwphysiodigestcom/files/ pdf / ACLpdf.

11. West RV and Harner CD. Graft selection in anterior cruciate ligament reconstruction. Journal of the American Academy of Orthopaedic Surgeons 2005; 13: 197-207.

12. Biau DJ, Katsahian S, Kartus J, et al. Patellar tendon versus hamstring tendon autografts for 
reconstructing the anterior cruciate ligament: a meta-analysis based on individual patient data. The American journal of sports medicine 2009; 37: 2470-2478.

13. Okoroha KR, Keller RA, Jung EK, et al. Pain Assessment After Anterior Cruciate Ligament Reconstruction: Bone-Patellar Tendon-Bone Versus Hamstring Tendon Autograft. Orthopaedic Journal of Sports Medicine 2016; 4: 2325967116674924.

14. Li S, Chen Y, Lin Z, et al. A systematic review of randomized controlled clinical trials comparing hamstring autografts versus bone-patellar tendon -bone autografts for the reconstruction of the anterior cruciate ligament. Archives of orthopaedic and trauma surgery 2012; 132: 12871297.

15. Abbas MM, Abulaban AA and Darwish HH. Functional outcomes of bone tendon bone versus soft tissue arthroscopic anterior cruciate ligament reconstruction. A comparative study. Saudi medical journal 2013; 34.

16. Spicer DD, Blagg SE, Unwin AJ, et al. Anterior knee symptoms after four-strand hamstring tendon anterior cruciate ligament reconstruction. Knee Surg Sports Traumatol Arthrosc 2000; 8: 286-289. DOI: 10.1007/s001670000139.

17. Shelbourne KD, Fu FH, Brown C, et al. The Anterior Cruciate Ligament: Reconstruction and Basic Science; Part Q - Complication; No 79 Anterior Knee Problems After ACL Reconstruction. Saunders, 2007. 